\title{
EFFECT OF CHARGE MODULATION ON THE ELECTROCONVECTIVE FLOW OF A LOW CONDUCTING LIQUID
}

\author{
Oleg O. Nekrasov AND Boris L. SMOROdin*
}

\begin{abstract}
Nonlinear evolution of the electroconvective flow patterns is analysed in a horizontal low conductive fluid layer under heating from above and under modulated charge injection. To examine the complex evolution of the system, numerical simulations are carried out using a finite difference method. The influence of amplitude and frequency modulation on the oscillatory electroconvection is studied. Traveling waves with modulated amplitudes and phase velocities and synchronously modulated patterns are found as stable solutions.
\end{abstract}

Mathematics Subject Classification. 76E25, 76E30,76E06, 76D55.

Received November 17, 2020. Accepted May 14, 2021.

\section{INTRODUCTION}

The behaviour of low conducting media (isotropic liquids or liquid crystals) in an electric field is of great interest to researchers due to the wide variety of nonlinear patterns observed in them $[4,5]$. The complexity of patterns is associated with various processes of charge generation and charge propagation in these media. In the case of charge injection a free charge is generated at the interface between the liquid and the electrode as a result of electrochemical redox reactions [23].

$$
M+\left(X^{+} Y^{-}\right) \rightarrow M(e)+\left(X Y^{-}\right) \rightarrow Y_{\text {free }}^{-}
$$

where $M$ is the metal of the electrode giving away the electron $e$ to the ion pair $\left(X^{+} Y^{-}\right)$. The ion $Y_{\text {free }}^{-}$is the injected charge, and the electrical conduction associated with this reaction is called the injection conduction. The reaction (1.1) is a classical example of unipolar injection (negative ions are formed at the cathode).

The existence of electroconvective flows in a plane capacitor filled with a low conducting liquid under of charge injection can be explained as follows. A negative charge appeared in a narrow near-cathode layer propagates to the bulk of the liquid under the action of the Coulomb forces. Moving in the capacitor gap charges may drag out the liquid, which leads to the instability and onset of an electroconvective flow. An analysis [8, 19] has shown that a steady electroconvection appears in the liquid as a result of backward bifurcation: two stable regimes (the conductive and the electroconvective) and a hysteresis transitions between them exist in a certain range of the control parameter.

Keywords and phrases: Electroconvection, injection, traveling wave, chage modulation.

Departmant of Physical Phase Transition, Perm State University, Perm 614990, Russia.

* Corresponding author: bsmorodin@yandex.ru 
The heating of the capacitor from above leads to growth of oscillatory disturbances [14, 18]. Stable traveling and modulated waves are found in this case $[11,18]$.

The instabilities and the nonlinear dynamics of convective systems can be strongly modified by external modulated driving forces, which can be used to control the fluid dynamics and pattern formations in various technological applications. The influence of modulated gravitational accelerations on the onset of RayleighBenard convection for the single component liquid was analysed in [6, 7]. It was shown that high-frequency vibrations, the period of which is much less than the characteristic times of the problem, can increase the stability of the quiescent state, while finite frequency vibrations can destabilize the quiescent state by a resonance phenomena. The effect of the temperature modulation on the convective threshold in a horizontal layer of a homogeneous fluid was investigated in [20,22]. The influence of temporal modulation in driving forces on pattern formation and nonlinear dynamics in pure liquids was investigated experimentally [1], and theoretically [2]. A Lorenz-like model of the hydrodynamic equations was used in these investigations.

The convection of a pure fluid under a modulated driving force can be related to two types of response: for a subharmonic response the oscillation period of the convective patterns is two times as large as the period of the external modulation; for a synchronous response the period of the latter coincides with the modulation period [6].

A more interesting situation appears in the case when in the absence of modulation oscillatory convection occurs.. Convection in binary mixtures [3] and non-uniform magnetic fluids [16], in systems with interfacial surfaces $[12,13]$, and electroconvection of nonisothermal liquid $[10,11,14,18]$ are an incomplete list of problems that manifest such behaviour. Moreover, several nonlinear modes that differ in their properties, such as the intensity of heat and mass transfer, usually coexist. A modulated driving force can be used to switch between convection modes to control transport processes in similar systems. In addition, under the influence of modulation, convective modes are transformed and new ones are observed. For example, quasi-periodic type of response appears. Quasiperiodic oscillations have two different characteristic frequencies which are not rationally related. In long convective cells or rings, quasi-periodic oscillations may correspond to modulated standing or traveling waves. Moreover, both amplitude and phase modulation are possible for traveling waves [17].

The purpose of the present paper is to describe the results of numerical simulations of electroconvection patterns in a low conducting liquid driven by both the steady temperature gradient and the charge modulation.

\section{GOVERNING EQUATIONS}

We consider a horizontal capacitor of thickness $d$ filled with a low conductive viscous liquid placed in the gravitational field $\mathbf{g}$. The liquid has the following properties: density $\rho_{0}$, kinematic viscosity $\tilde{\nu}$, thermal diffusivity $\chi$, permittivity $\varepsilon$. We assume that all transfer coefficients and charge mobility $K$ are constants. The potential difference $V_{0}$ and temperature difference $\Theta$ are applied to the plates of the capacitor. The temperature gradient is opposite to the direction of the gravitational field. Mathematically, this problem is equivalent to the problem of a horizontal layer bounded by rigid, perfectly heat- and electric-conducting boundaries located at $z=0$ and $z=d$. Negative charges are injected at the lower boundary (cathode). We use the system of coordinates with the $\mathrm{x}$ axis directed along the lower plate of the capacitor and the $\mathrm{z}$ axis perpendicular to the layer (Fig. 1). We use the following equation of state for an inhomogeneously heated liquid

$$
\rho=\rho_{0}(1-\beta \theta)
$$

where the thermal expansion coefficient $\beta=\frac{1}{\rho_{0}}\left(\frac{\partial \rho}{\partial \theta}\right)>0, \theta$ is deviation of temperature from its average value $\bar{\theta}$.

The electroconvection of a nonisothermal incompressible liquid placed into the gravitational and electric fields $[9,11,19]$ is described in the Boussinesq approximation by the set of equations which includes the Navier-Stokes equation, the heat conductivity equation, the charge conservation law, the continuity equation and the Poisson 


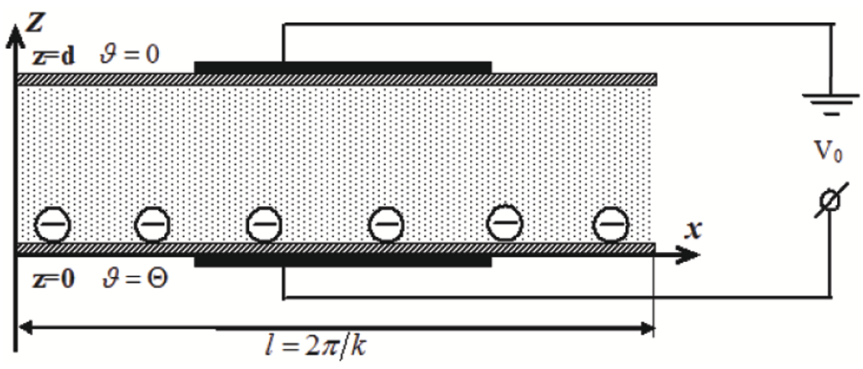

Figure 1. Problem geometry and coordinate system.

equation:

$$
\begin{aligned}
& \frac{\partial \vec{v}}{\partial t}+(\vec{v} \cdot \nabla) \vec{v}=-\frac{1}{\rho_{0}} \nabla p+\tilde{\nu} \Delta \vec{v}+g \beta \theta \vec{e}-q \nabla \Phi \\
& \frac{\partial \theta}{\partial t}+(\vec{v} \cdot \nabla) \theta=\chi \Delta \theta \\
& \frac{\partial q}{\partial t}+(\vec{v} \cdot \nabla) q=K\left(q^{2}-\nabla \Phi \cdot \nabla q\right) \\
& \operatorname{div} \vec{v}=0 \\
& \frac{q}{\varepsilon_{0} \varepsilon}=-\Delta \Phi
\end{aligned}
$$

here $\vec{v}, p, \theta$ - velocity, pressure and temperature, $q$ is charge density, $\Phi$ is electrical potential, $\vec{e}=(0,0,1)-$ unit vector, $\varepsilon_{0}$ - electric constant.

The external forces acting on the unit volume of the liquid include i) the electric (Coulomb) force that characterizes the action of the electric field on the charge of the medium and ii) the buoyancy force. The charge flux includes mobility of the charge in the electric field and the convective transfer.

We consider the case when the injection at the cathode is proportional to the electric field strength $z=0: q=a(t) E$ (E is electric field strength, and the modulation $a(t)=a(1+\alpha \sin (2 \pi f t)$ ) with amplitude $\alpha$ and frequency $f$ is provided by oscillation of the potential on an ion-selective membrane [15] (or a grid electrode) near the cathode.

We apply the following scales: length $[x]=d$, time $[t]=d^{2} / \tilde{\nu}$, velocity $[v]=\tilde{\nu} / d$, temperature $[\theta]=\Theta$, potential difference $[\Phi]=V_{0}$, charge density $[q]=\varepsilon_{0} \varepsilon V_{0} / d^{2}$ and $[p]=\rho_{0} \tilde{\nu}^{2} / d^{2}$.

$$
\begin{aligned}
& \frac{\partial \vec{v}}{\partial t}+(\vec{v} \cdot \nabla) \vec{v}=-\nabla p+\Delta \vec{v}+\frac{\operatorname{Ra}}{\operatorname{Pr}} \theta \vec{e}-\frac{T^{2}}{M^{2}} q \nabla \Phi, \\
& \frac{\partial \theta}{\partial t}+(\vec{v} \cdot \nabla) \theta=\frac{1}{\operatorname{Pr}} \Delta \theta, \\
& \frac{\partial q}{\partial t}+(\vec{v} \cdot \nabla) q=\frac{T}{M^{2}}\left(q^{2}-\nabla \Phi \cdot \nabla q\right), \\
& \operatorname{div} \vec{v}=0, \\
& \Delta \Phi=-q .
\end{aligned}
$$

In the case of impenetrable rigid and perfectly heat- and electroconducting boundaries of the layer, we have the following boundary conditions:

$$
\begin{aligned}
& z=0: \vec{v}=0, \theta=1, \Phi=0, q=A(1+\alpha \sin 2 \pi \nu t) E \\
& z=1: \vec{v}=0, \theta=0, \Phi=1 .
\end{aligned}
$$


In addition to hydrodynamic dimensionless parameters, such as the Rayleigh number $\mathrm{Ra}=g \beta \Theta d^{3} / \tilde{\nu} \chi$ and the Prandtl number $\operatorname{Pr}=\tilde{\nu} / \chi$, system of equations (2.11) and boundary conditions (2.12) contain dimensionless electric parameter $T=\varepsilon_{0} \varepsilon V_{0} \rho_{0} / K \nu$, dimensionless frequency of modulation $\nu=f d^{2} / \tilde{\nu} . A=a d / \varepsilon_{0} \varepsilon$ and $M=$ $\sqrt{\varepsilon_{0} \varepsilon / K^{2} \rho_{0}}$ characterize the intensity of charge injection and the mobility of injected charges respectively. Depending on the type of the medium and charge carriers, parameter $M$ can vary over wide range $4<M<120$ [8].

\section{Solution method And Diagnostic TOOL}

To analyse two-dimensional nonlinear electroconvective flows of the low conducting liquid, we used the method of solution, in which velocity components are replaced with two scalar functions (fields) to exclude pressure. For this purpose, we introduce the stream function field $\psi$ and the vorticity field $\varphi$, which are velocity related as:

$$
v_{x}=-\frac{\partial \psi}{\partial z}, v_{z}=\frac{\partial \psi}{\partial x}, \varphi=(\operatorname{rot} \vec{v})_{y}
$$

In the case of weak injection $(A \ll 1)$, the nonlinear problem can be solved in the non-inductive approximation, in which the electric field in the layer is independent of the redistribution of charges due to convective motion $[11,21]$. In case of the steady injection a comparison of the results of solving the problem in the complete formulation and in the non-inductive approximation has shown that the results differ by less than $1 \%$ [11].

$$
\begin{aligned}
& \frac{\partial \varphi}{\partial t}-\frac{\partial \varphi}{\partial x} \frac{\partial \psi}{\partial z}+\frac{\partial \varphi}{\partial z} \frac{\partial \psi}{\partial x}=\Delta \varphi-\frac{T^{2}}{M^{2}} E_{z} \frac{\partial q}{\partial x}-\frac{\operatorname{Ra}}{\operatorname{Pr}} \frac{\partial \theta}{\partial x} \\
& \frac{\partial \theta}{\partial t}-\frac{\partial \theta}{\partial x} \frac{\partial \psi}{\partial z}+\frac{\partial \theta}{\partial z} \frac{\partial \psi}{\partial x}=\frac{1}{\operatorname{Pr}} \Delta \theta \\
& \frac{\partial q}{\partial t}-\frac{\partial q}{\partial x} \frac{\partial \psi}{\partial z}+\frac{\partial q}{\partial z} \frac{\partial \psi}{\partial x}=\frac{T}{M^{2}}\left(q^{2}+E_{z} \frac{\partial q}{\partial z}\right), \\
& \varphi=-\Delta \psi \\
& E_{z}=-\frac{3 A(1+2 A z)^{1 / 2}}{1-(1+2 A)^{1 / 2}} .
\end{aligned}
$$

Here $E_{z}$ is electric field strength in the absence of modulation and convective flows. The conditions imposed on the horizontal boundaries describe the absence of slip along them, fixed temperature and potential and modulated charge on the cathode:

$$
\begin{aligned}
& z=0: \psi=0, \frac{\partial \psi}{\partial z}=0, \theta=1, q=-A(1+\alpha \sin (2 \pi \nu t)) E_{z} \\
& z=1: \psi=0, \frac{\partial \psi}{\partial z}=0, \theta=0
\end{aligned}
$$

Periodic boundary conditions, $\bar{f}(x, z, t)=\bar{f}(x+\lambda, z, t)$, imposed on the lateral sides of a convection cell, are assigned to all variable fields $\bar{f}=(\psi, \varphi, \theta, q)$ with $\lambda=2$.

To solve the system of equations (3.2)-(3.8) the finite-difference method is applied. The vorticity equation (3.2) is solved using the explicit algorithm. Switching between the algorithm with central differences and the algorithm with upwind scheme is performed depending on the stability criterion. The Poisson equation (3.5) that connects the stream function with the vorticity is solved by means of an iterative method of successive over-relaxation at each time step. To find the solutions to the charge and heat-transfer equations, we use the explicit algorithm with upwind scheme. All calculations are executed on a grid of 41x21 nodes. A further mesh refinement did not provide a significant improvement in the evaluation of solution characteristics. 
The numerical algorithm used in our investigation was tested in the case of steady electroconvection of isothermal liquid [21] $(\mathrm{A}=0.25, \mathrm{M}=14.14, \mathrm{Pr}=10$ and $\mathrm{L}=2)$. As expected, the steady electroconvection appears as result of backward bifurcation at $T_{1}=6280$. Two stable solutions (steady convection and mechanical equilibrium) coexist in the range $T_{2}<T<T_{1}$. The steady convection disappears and a transition to equilibrium take placein $T_{2}=1780$. Our results are in good agreement with the results obtained in [21] $T_{1}=6200$ and $T_{2}=1800$.

To monitor the electroconvective flows, the following characteristics are analysed:

(1) the maximum anf minimum values of the stream function in the in the $x-z$ plane

$$
\psi_{\max }(t)=\max _{x, z} \psi(x, z, t), \quad \psi_{\min }(t)=\min _{x, z} \psi(x, z, t)
$$

(2) the value of the stream function in the reference point $x_{0}, z_{0}$ of the convection cell

$$
\psi_{\mathrm{loc}}(t)=\psi\left(x_{0}=\lambda / 4, z_{0}=1 / 2, t\right),
$$

(3) the lateral phase velocity $v_{p h}$, which is measured by the time derivative of $x_{\psi}$, where $x_{\psi}-$ maximum locations of $\psi(x, z=1 / 2, t)$ at the midheight of the liquid layer $z=1 / 2$ :

$$
v_{p h}=\frac{\mathrm{d} x_{\psi}}{\mathrm{d} t} .
$$

To elucidate more detailed information on the electroconvective flows and the spatiotemporal complexity of the liquid behaviour we have also studied lateral Fourier decompositions,

$$
\tilde{f}(x, t)=\tilde{f}_{0}(t)+\operatorname{Re}\left[\sum_{n=1}^{\infty} \hat{\tilde{f}}_{n}(t) e^{-i n k x}\right]
$$

of the stream function, charge, and temperature fields at midheight position, $z=1 / 2$. The behavior there is largely representative for all other $z$.

The oscillatory electroconvection is characterised by the stream function, the temperature, the charge oscillation at a fixed point of the convective cell. The frequency characteristic of oscillations can be obtained by means of the temporal Fourier analysis of the stream function at a fixed point $\psi_{\text {loc }}(t)$.

\section{Steady injection}

Let us consider the nonlinear evolution of elctroconvective patterns in a low conductive liquid layer heated from above. In calculations, we employed the following parameters: $\operatorname{Pr}=10, M=14.14$ [8, 11, 14], $\operatorname{Ra}=-2500$ and $A=0.25$ which are correspond to the case of oscillatory instability.

The electroconvection of a low conducting liquid arises as a forward Hopf bifurcation at the electric parameter $T>T=6778$ (Fig. 2, a) and the traveling wave (TW) is formed as a result of a transient process. The snapshots of the stream function and the charge at $T=6800$ in the TW solution are shown in Figure $2 \mathrm{~b}$ and c. A small flow intensity only slightly distorts the charge contour lines. Deviations of the charge from its equilibrium value are well described by the first spatial harmonic. Although the right and left directions of the wave propagation are equally possible, but in our consideration traveling wave moves from right to left. The value of the stream function at a fixed point $\psi_{\text {loc }}$ oscillates with the fundamental frequency $\nu_{0}=1$. . This value of the dimensionless frequency $\nu_{0}$ corresponds to the modulation frequency $f=2.1 \mathrm{hz}$ in the case of ethanol with kinematic viscosity $1.5 \times 10^{-6} \mathrm{~m}^{2} / \mathrm{c}$ in a capacitor with a distance between the plates of $1 \mathrm{~mm}$.

In the interval $6816<T<6821$, traveling waves lose their stability (Fig. 2a) and the regime of modulated standing waves (MSWs) is formed in the layer, which are characterized by the amplitude modulation in the 

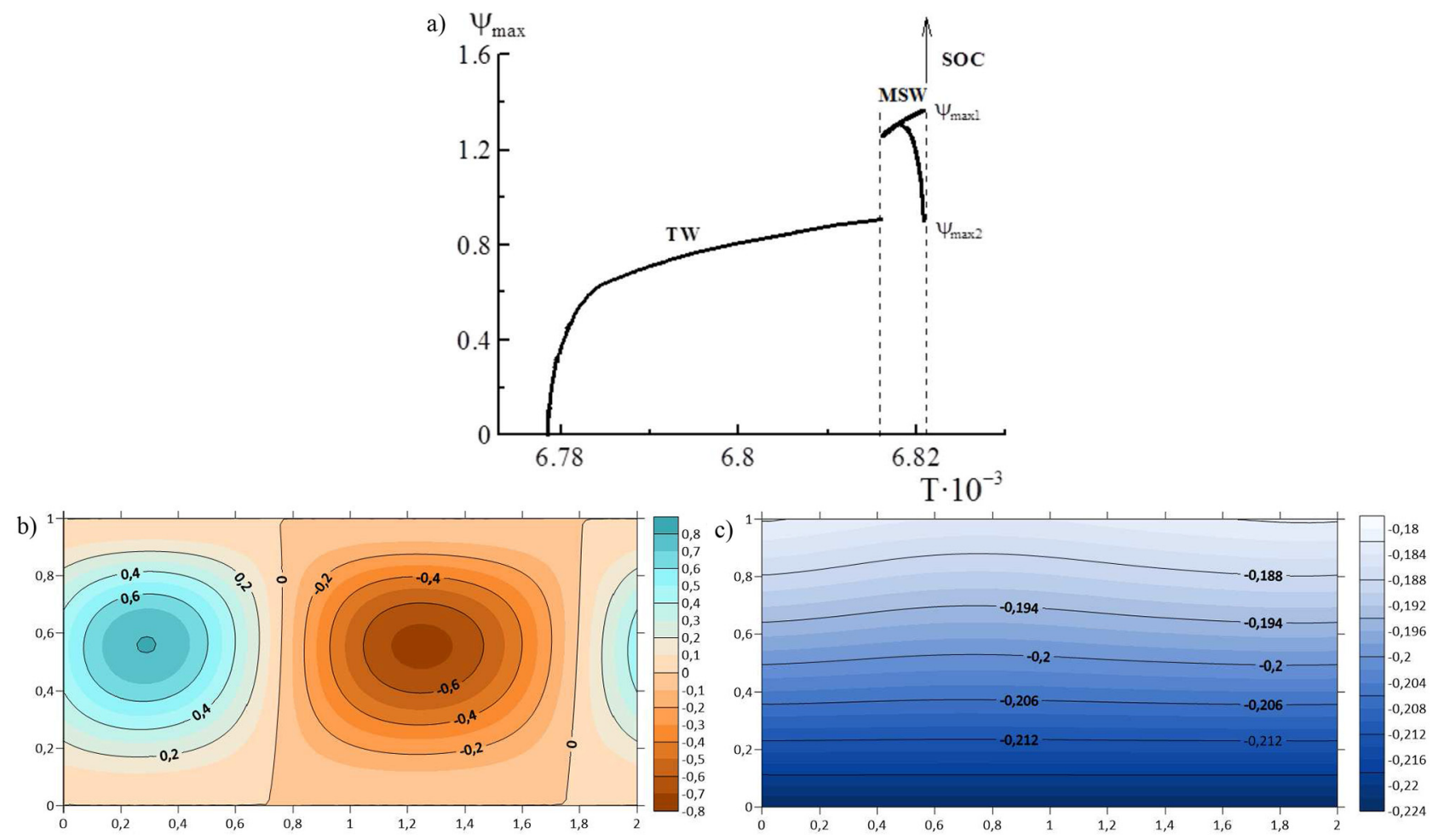

FiguRE 2. Steady (non-modulated) injection. (a) The bifurcation map of laterally extended electroconvective states in a low conductive liquid as functions of the electrical parameter $T$; (b) the structure of the stream function and the charge in the traveling wave regime (TW, $T=6800)$. Parameters are $\operatorname{Pr}=10, M=14.14, \mathrm{Ra}=-2500$.

interval $\left[\psi_{\max 1}, \psi_{\max 2}\right]$. The lateral motion of the liquid as a whole along the $x$ axis is absent. Note that the intensity of convective motion in the regimes of modulated standing (MSW) or traveling waves (TW) is relatively weak $\psi_{\max }<1.4$ (Fig. 2).

A further increase in parameter $T$, the intensity of the electroconvective flow sharply increases, and the system transfers to the regime of steady overturning convection (SOC in Fig. 2, the transition is marked with an up arrow), in which maximal value of the stream function $\psi_{\max }=26$ is much greater than in the traveling wave mode. This solution is not shown in the bifurcation map.

\section{Modulated inJeCtion}

The nonlinear response of an electroconvective system depends on the amplitude and frequency of charge modulation at the cathode. Let's consider the stable solutions at different frequencies. At a modulation frequency $\nu$ that is not a multiple of the fundamental frequency $\nu_{0}$, the modulated traveling wave is observed. The behaviour of the various flow characteristics: $\psi_{\text {loc }}, \psi_{\max }, \psi_{\min }$, versus time and Fourier-spectra, at the modulation amplitude $\alpha=0.05$ are shown Figure 3. Generally, the oscillations of the stream function at a fixed point are quasi-periodic. The Fourier spectrum of this oscillation shown in Figure $3 \mathrm{~b}$ contains few contributions. They are located at the combinations of the fundamental frequency of traveling wave in unmodulated case $\nu_{0}$ and the modulation frequency $\nu$. The largest peak corresponds to $\nu_{0}$. The height of the peaks at $\nu_{0} \pm \nu$ in the spectrum are smaller. Higher Fourier harmonics in the evolution of $\psi_{\text {loc }}$ are very small. 

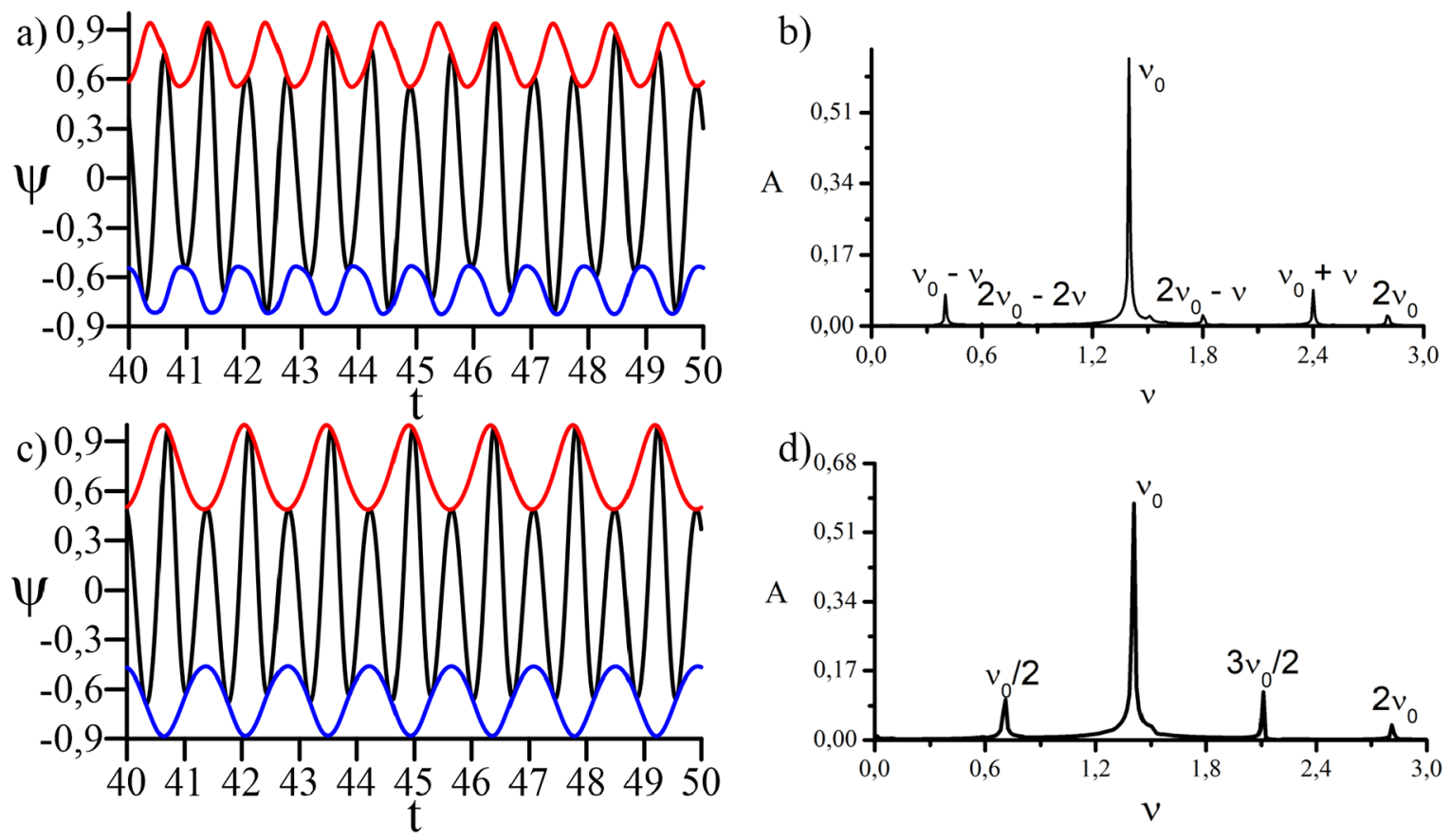

Figure 3. The oscillations of the stream function $\psi_{\text {loc }}$ at a fixed point (black lines) and its envelopes (red lines for $\psi_{\max }$ and blue lines for $\psi_{\min }$ ) at $(a) \nu=1$, and $(c) \nu=0.7$; the Fourier spectra of $\psi_{\text {loc }}(b) \nu=1$, and $(d) \nu=0.7$. Parameters are $\operatorname{Pr}=10, M=14.14, \operatorname{Ra}=-2500$, $T=6800$.

In the case when the modulation frequency $\nu=\nu_{0} / 2=0.7$ oscillations of the stream function at a fixed point $\psi_{\text {loc }}$ (Fig. 3c) contain only multiples of the modulation frequency $\nu=\nu_{0} m$, where $m$ is an integer (Fig. 3d).

At modulation frequencies $(\nu=0.7, \nu=1.0)$ traveling waves are strongly modulated in amplitude, but weakly modulated in phase. This amplitude modulated traveling wave (TW A) has $v_{p h} \approx\left\langle v_{p h}\right\rangle$. Qualitatively, the snapshots of the stream and charge functions are similar to those that characterize a traveling wave in the stationary case (Fig. $2 \mathrm{~b}$ and c). The amplitude of the stream function in a convective cell and hence the intensity of the electroconvection oscillates between envelopes $\psi_{\max }(t)$ (red lines in Fig. 3 ) and $\psi_{\min }(t)$ (blue lines in Fig. 3).

The behavior of a electroconvective flow can change dramatically when the modulation frequency is twice as large as the fundamental frequency of the system $\nu=2 \nu_{0}$ (Fig. 4). Instead of the expected subharmonic response a different type of solution appears with phase fixed modulated electroconvection (MEC) that oscillates synchronously with the modulated injection. The transition from the traveling wave solution to the modulated electroconvection mode is shown in Figure 4 for different values of the modulation amplitude. The traveling wave remains stable only under the low-amplitude modulation $\alpha=0.005$. For large values of modulation amplitudes, the traveling wave loses stability, and a more intense convection mode is established in the system. As one can see from Figure 4 an increase in the amplitude leads to a decrease in the transient time. Figure 5 shows snapshots of the stream function, charge and temperature distribution in the case of modulated convection.

These fields look like the one in SOC states under stationary driving with the mirror symmetry between oppositely turning rolls and with the electroconvective mixing being much stronger than for TWs.

The calculations have shown that charge modulation with amplitude $\alpha=0.05$ and frequency $\nu=2 \nu_{0}$ destroys the traveling wave mode for any values of the parameter $T(6778<T<6816)$, at which TW existed under steady injection. 


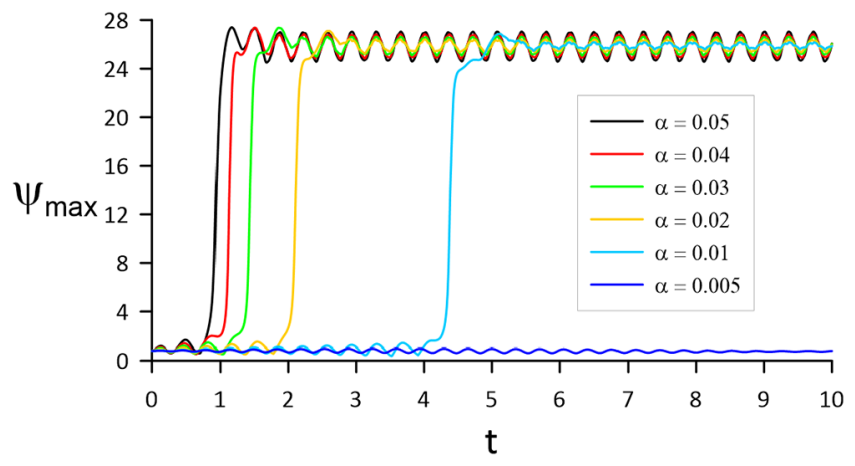

Figure 4. The temporal evolution of the stream function amplitude $\psi_{\max }$ in modulated electroconvection (MEC). Parameters are $M=14.14, \mathrm{Ra}=-2500$.
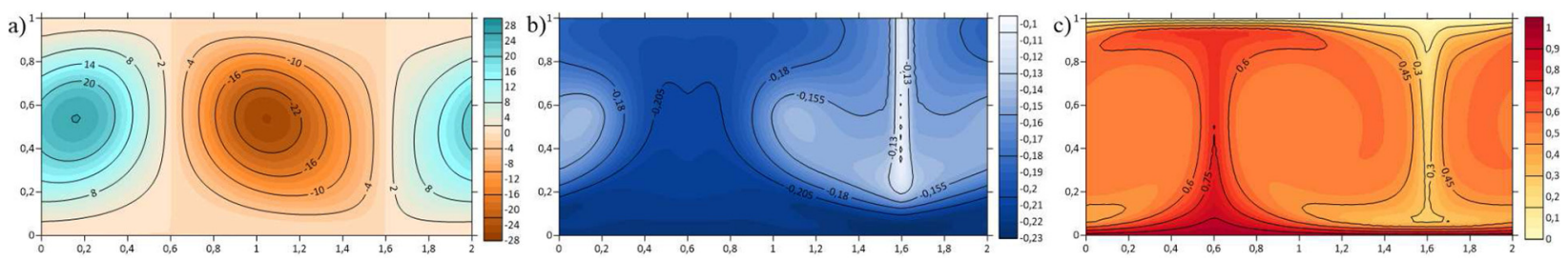

Figure 5. Snapshots of (a) the stream function; (b) the charge; and (c) temperature distributions for phase fixed modulated electroconvection. Parameters are $\operatorname{Pr}=10, M=14.14$, $\mathrm{Ra}=-2500, T=6800, \alpha=0.05$.

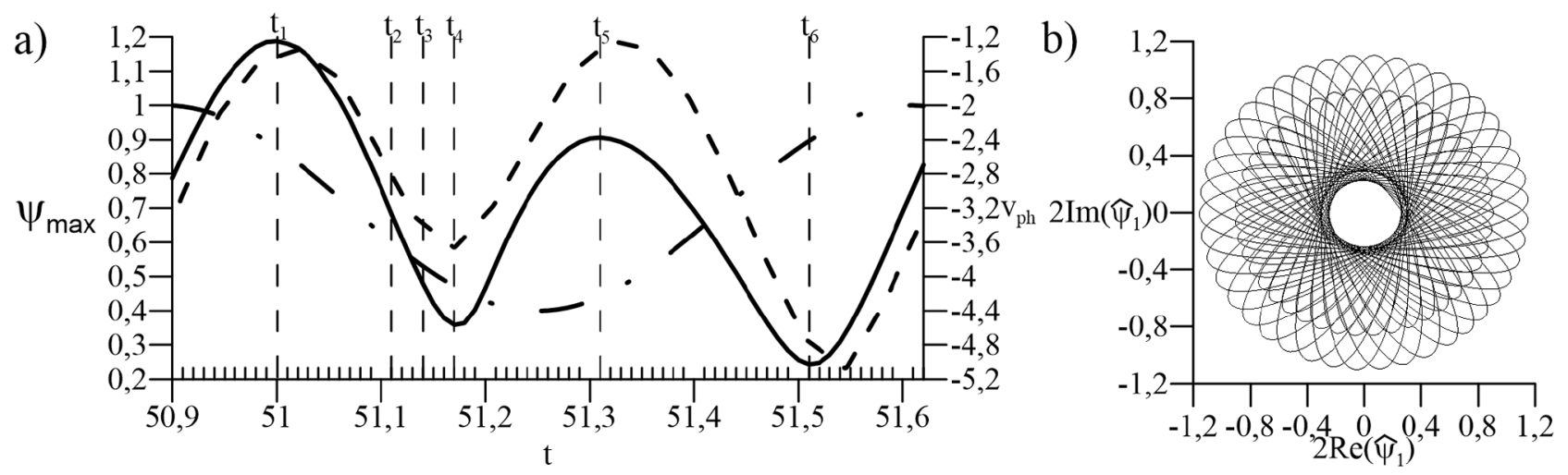

Figure 6. (a) The dependencies of the maximum value of the stream function on time (solid line) and the phase velocity (dashed line) and a schematic representation of the charge injection (dashed dotted line); (b) the trajectories of the first lateral Fourier mode $\hat{\psi}_{1}(t)$. Parameters are $\operatorname{Pr}=10, M=14.14, \mathrm{Ra}=-2500, T=6800, \nu=\nu_{0}=1.4, \alpha=0.05$.

Finally we discuss the case when the modulation frequency coincides with the fundamental frequency of the system $\nu=\nu_{0}(\alpha=0.05)$. In this case, linear stability analysis predicts the synchronous response of the system to external influences $[6,20,22]$. In the non-linear case, more complex solutions, such as quasi-periodic and chaotic oscillations, may arise in supercritical region. In our case, there are two main frequencies in the Fourier spectrum of the quasi-periodic oscillations of the stream function at a fixed point: $\nu_{1}=1.367$ and $\nu_{2}=1.442$, 

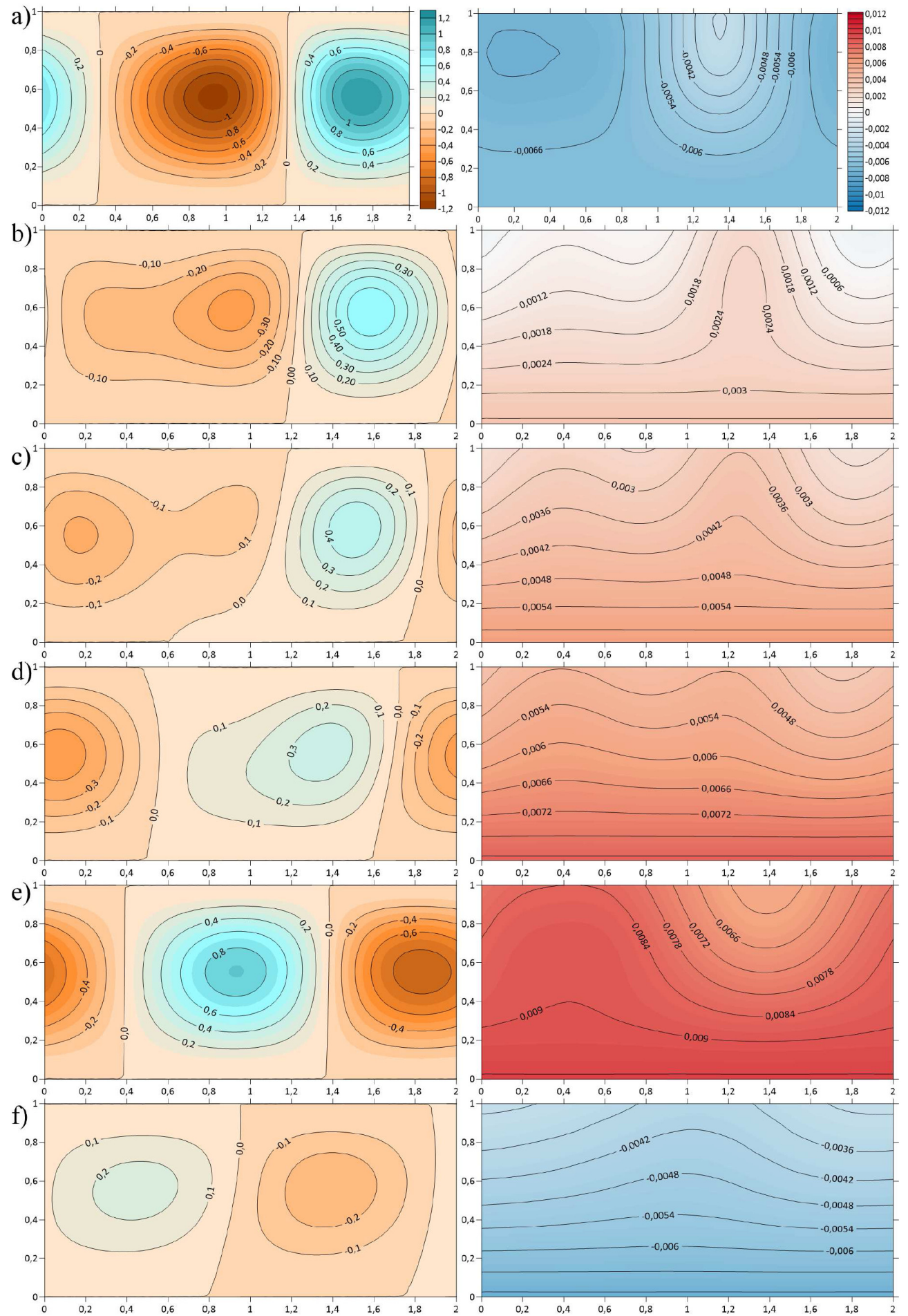

FIgURE 7. Snapshots of the stream function $\psi$ (the left column) and charge deviation $\delta q$ (the right column) in the phase modulated TW $\nu=\nu_{0}=1.4$. The times from top to bottom, are indicated by vertical dashed lines in Figure 6. Parameters are $\operatorname{Pr}=10, M=14.14, \mathrm{Ra}=-2500$, $T=6800, \alpha=0.05$. 
and modulated both by phase and amplitude traveling wave (TW B) is generated under modulation with an amplitude $\alpha=0.05$.

To elucidate the consequences of this solution we show in Figure 6 a schematic representation of the charge injection, $\psi_{\max }$, and the phase velocity $v_{p h}$ of the TW B over modulation period as well as the trajectories of the first lateral Fourier mode $\hat{\psi}_{1}(t)$ of the stream-function $\psi(x, z=1 / 2, t)$ in the plane spanned by the real and imaginary part of $\hat{\psi}_{1}(t)$. The snapshots of the stream function corresponding to different time points in the charge modulation period are shown in Figure 7. In the color online version the vertical color bars (Fig. 7a) show the coding of the stream function and the charge deviations in all moments of time $t_{1}-t_{6}$.

The common scalar bars used for all distributions are shown in Figure 7. In contrast to the MEC solution the mirror symmetry between oppositely turning rolls does not exist. The $\psi_{\max }$ and $v_{p h}$ oscillate with the period $T=2 / \nu$ of the driving. Note the large amplitude oscillations of $v_{p h}$ and the complex variation of the stream function (Fig. 7). They show already that the flow dynamics of amplitude and phase modulated TW B is significantly more varying and more complex than those of amplitude modulated TWs A at $\left(\nu<\nu_{0}\right)$.

We describe in detail the evolution of the stream function $\psi(x, z, t)$ and deviations of the charge from their distribution in the quiescent liquid under steady injection $\delta q(x, z, t)=q(x, z, t)-q_{\text {cond }}(z)$ in a traveling wave TW B. The highest phase velocity values approximately correspond to the minimum of the stream function amplitude $\psi_{\max }$. $\psi$ is proportional to the vertical component of velocity therefore fast lateral movement of the TW leads to weak convective mixing.

The negative deviations of the charge $\delta q(x, z)$ at $t_{1}$ change to the positive one in the range $t_{2}-t_{4}$. The absolute value of phase velocity $v_{p h}$ growths.

At $t_{1}$ (Fig. 7a) rotation intensity of neighbouring rolls are maximal and approximately the same. In the range $t_{1}-t_{4}$ (Fig. 6a), the amplitude of the traveling wave $\psi_{\max }$ decrease following a decrease in the charge injected from the electrode. In the interval $t_{1}-t_{3}(\mathrm{a}-\mathrm{c})$ in Figure 7 clockwise turning vortex has a greater intensity than the opposite one. In the same time the anticlockwise turning vortex transforms into two ones of the same turning. This behaviour and the corresponding behaviour of the charge deviation are explained by the generation of higher lateral harmonics. In the range $t_{4}-t_{5}$ (Fig. 6a) the convective mixing increases and riches local maximum at $t_{5}$, and the TW $\mathrm{B}$ with distributions (Fig. 7e) moves the most slowly. In the interval $t_{5}-t_{6}$ (Fig. 6a) the convective mixing decreases and lateral movement accelerates, the positive deviations of the charge $\delta q(x, z)$ at $t_{5}$ change to the negative one (Fig. $7 \mathrm{f}$ ).

To elucidate in detail the spatiotemporal complexity of the TW B considered above we used also the lateral Fourier decomposition of the stream function, in particular at midheight position $z=1 / 2$. In contrast to the charge field the lateral variation of $\psi$ is largely harmonic, so that the first Fourier mode $\hat{\psi}_{1}(t)$ in representation (Fig. 6b) characterizes the flow behaviour. The trajectory of $\hat{\psi}_{1}(t)$ is the projection of the spatiotemporal flow dynamics of the convective state in real space into lateral Fourier space. It complements the picture of the TW dynamics of $\psi$ at a fixed location. In (Fig. $6 \mathrm{~b}$ ) the time evolution of the complex mode $\hat{\psi}_{1}(t)$ is presented in the plane spanned by its real and imaginary parts. There we show the time interval of 37 modulation periods. The motion of $\hat{\psi}_{1}(t)$ is limited by two circles. In this time $\hat{\psi}_{1}(t)$ moves 37 times from the inner circle with radius $\hat{\psi}_{1}=0.24$ to the outer circle with axes $\hat{\psi}_{2}=1.19$ as a result of the amplitude modulation.

\section{Conclusion}

The spatiotemporal behaviour of oscillating electroconvection rolls in low conductive liquid subject to sinusoidal modulation of the cathode's charge and heating from above have been analysed with finite difference numerical simulations. Various diagnostic tools have been used to elucidate the spatiotemporal structure of the rich and rather complex, strongly nonlinear response behaviour to modulation of the charge driving. With charge modulation the Coulomb induced advection gets modulated and leads in particular for the modulated TWs to complex nonlinear mixing behaviour.

It is shown that charge modulation can be used to control flow dynamics with various properties. We found the following different response characteristics depending on $\nu$ : (i) Stable SOC states occuring for $\alpha=0$ at 
$\nu=2 \nu_{0}$ become synchronously modulated and oscillate with fixed phase around the SOC solution. (ii) The amplitude modulated TWs has modulation of their amplitude thus becoming quasiperiodic states. (iii) The TW B, which velocity $v_{p h}$ becomes modulated at $\nu=\nu_{0}$. Here, the behaviour of the stream function and the charge are very complex with generation of highest lateral harmonics.

\section{REFERENCES}

[1] G. Ahlers, P.C. Hohenberg and M. Lücke, Thermal convection under external modulation of the driving force. II. Experiments. Phys. Rev. A 32 (1985) 3519-3534.

[2] G. Ahlers, P.C. Hohenberg and M. Lücke, Thermal convection under external modulation of the driving force. I. The Lorenz model. Phys. Rev. A 32 (1985) 3493-3518.

[3] W. Barten, M. Lücke, W. Hort and M. Kamps, Fully developed traveling-wave convection in binary fluid mixtures. Phys. Rev. Lett. 63 (1992) 376-379.

[4] A. Buka and L. Kramer, Pattern Formation in Liquid Crystals. Springer, New York (1995).

[5] M.C. Cross and P.C. Hohenberg, Pattern formation outside of equilibrium. Rev. Mod. Phys. 65 (1979) 851-1112.

[6] G.Z. Gershuni and E.M. Zhukhovitsky, Convective Stability of Incompressible Fluids. Keter, Jerusalem (1976).

[7] P.M. Gresho and R.L. Sani, The effects of gravity modulation on the stability of a heated fluid layer. J. Fluid Mech. 40 (1970) $783-806$.

[8] J.C. Lacroix, P. Atten and E.J. Hopfinger, Electroconvection in a dielectric liquid layer subjected to unipolar injection. J. Fluid Mech. 69 (1975) 539-563.

[9] L.D. Landau and E.M. Lifschitz, Vol. 6 of Course of Theoretical Physics. Pergamon, Oxford, Reading, MA (1993).

[10] T.F. Li, X.R. He, K. Luo and H.L. Yi, Oscillatory flows of electro-thermo-convection in eccentric annulus. Int. J. Heat Mass Transfer 134 (2019) 920-932.

[11] A.N. Mordvinov and B.L. Smorodin, Electroconvection under injection from cathode and heating from above. J. Exp. Theor. Phys. 114 (2012) 870-877.

[12] A. Nepomnyashchy and I. Symanovskii, Generation of nonlinear Marangoni waves in a two-layer film by heating modulation. J. Fluid Mech. 771 (2015) 159-192.

[13] A. Nepomnyashchy, I. Symanovskii, A. Viviani and F. Dubois, The action of temporal modulation of an interfacial heat consumption on nonlinear Marangoni waves in the presence of gravity. Eur. J. Mech. B 84 (2020) 51-62.

[14] F. Pontiga and A. Castellanos, Physical mechanisms of instability in a liquid layer subjected to an electric field and a thermal gradient. Phys. Fluids 6 (1994) 1684-1701.

[15] I.I. Ryzhkov, A.S. Vyatkin and M.I. Medvedeva, Modelling of electrochemically switchable ion transport in nanoporous membranes with conductive surface. J. Siberian Federal Univ. Math. Phys. 12 (2019) 579-589.

[16] M.I. Shliomis, B.L. Smorodin and S. Kamiyama, The onset of thermomagnetic convection in stratified ferrofluids. Philos. Mag. 83 (2003) 2139-2153.

[17] B.L. Smorodin and M. Lücke, Convection in binary fluid mixtures with modulated heating. Phys. Rev. E 79 (2009) 026315.

[18] A.V. Taraut and B.L. Smorodin, Electroconvection in the presence of steady unipolar injection and residual conductivity. J. Exp. Theor. Phys. 115 (2012) 361-369.

[19] Ph. Traore, A.T. Perez, D. Koulova and H.J. Romat, Numerical modelling of finite-amplitude electro-thermo-convection in a dielectric liquid layer subjected to both unipolar injection and temperature gradient. J. Fluid Mech. 658 (2010) $279-293$.

[20] G. Venezian, Effect of modulation on the onset of thermal convection. J. Fluid Mech. 35 (1969) 243-254.

[21] A.N. Vereshchaga and E.L. Tarunin, in Numerical and Experimental Simulation of Hydrodynamic Phenomena under Weightlessness, edited by V.A. Briskman (Ural Branch of the Academy of Sciences of the Soviet Union, Sverdlovsk, 1988), p. 93 [in Russian].

[22] C.S. Yin and C.H. Li, Instability of unsteady flows or configurations. Part 2. Convective instability. J. Fluid Mech. 54 (1972) $143-152$.

[23] A.I. Zhakin, Near-electrode and transient processes in liquid dielectrics. Phys.—Usp. 49 (2006) $275-295$. 